\title{
Über die Spaltung der Hefenucleinsäure durch Bakterien.
}

\author{
II. Mitteilung.
}

Von

\author{
A. Schittenhelm und F. Schröter. \\ Mit vier Kurvenzeichnungen. \\ (Aus der med. Klinik der Universität Breslau.) \\ (Der Redaktion zugegangen am 23. Sept. 1903.)
}

In unserer ersten Mitteilung ${ }^{1}$ ) haben wir nachgewiesen, daß durch die Einwirkung von Mikroorganismen eine Abspaltung von Nucleinbasen aus Hefenucleinsäure stattfindet.

Unmittelbar vor deren Drucklegung erschien von Iw a n off ${ }^{2}$ ) eine Arbeit, in welcher er eine fermentative Zersetzung der Thymonucleinsäure durch Schimmelpilze unter Abspaltung von freien Nucleinbasen beschrieb.

In unseren Versuchen hatten wir lediglich mit Reinkulturen von Bacterium coli gearbeitet. Es schien uns daher für unsere. ferneren Untersuchungen erwünscht, ein Urteil darüber zu gewinnen, ob sich die verschiedenen Bakterienarten in ihrer Wirksamkeit bezïglich der Spaltung von Hefenucleinsäure unterscheiden und welche von ihnen am intensivsten einwirken. Indem wir also den Zweck verfolgten, die günstigsten Bedingungen für eine möglichst eingreifende Zersetzung und entsprechend vermehrte Ausbeute zu finden, haben wir neben Reinkulturen auch Gemische von Bakterien untersucht. Von unserem ursprünglichen Plan, die Untersuchungen auf eine größere Menge der verschiedensten Bakterienarten auszudehnen, um eventuell differentialdiagnostische Merkmale aufzufinden, glaubten wir für jetzt um so eher Abstand nehmen zu können,

1) Diese Zeitschr., Bd. XXXIX, S. 203 ff.

2) Diese Zeitschr., Bd. XXXIX, S. 31. 
als wir aus der Mitteilung von Plenge ${ }^{1}$ ) ersehen, daß dieser sich mit Untersuchungen über die differentialdiagnostische Verwendbarkeit der Nucleinsäure beschäftigt. Wir beschränkten uns daher auf Versuche mit Bact. coli, Staphylococcus und einem Gemisch von Fäulnisbakterien, das durch Aufschlämmen kleiner Stückchen ganz frischer Faeces in sterilem Wasser gewonnen wurde.

Als Nährlösung kam für diese Versuche die in Mitteilung I näher beschriebene Uschinskysche Flüssigkeit in Anwendung, worin wir die $\mathrm{N}$-haltigen Bestandteile durch hefenucleinsaures Natron ersetzten. Die Verarbeitung der Reaktionsprodukte wurde in der Weise verteilt, daß wir für alle Bakterienarten mehrere Versuche ansetzten, die in Abständen von je 5 Tagen untersucht wurden. Wir beabsichtigten damit, ungefähr festzustellen, wie die "Reaktionskurven» sich gestalten würden.

\section{Bacterium coli.}

Versuch I. $1500 \mathrm{ccm}$ Uschinskysche Lösung (sine natrium asparaginic. und ammon.lactic.) $+15 \mathrm{~g}$ nuleinsaures Natrium mit frisch gezüchteter Colireinkultur geimpft und in 3 Portionen à $500 \mathrm{ccm}$ in den Brutschrank gestellt. Vor der Verarbeitung wurde nochmals darauf geprüft, ob die aufgegangene Kultur ihre Reinheit bewahrt hatte.

Portion 1 wurde nach 5 Tagen herausgenommen, durch ein Bakterienfilter ${ }^{2}$ ) gesaugt und im Filtrat die freigewordenen Purinbasen mit Hilfe der Fällung durch ammoniakalische Silberlösung herausgeholt.

Gefunden: 1,4 mg Gesamtbasen-N.

1) Diese Zeitschr., Bd. XXXIX, S. 180.

2) Als Bakterienfilter benutzten wir Büchnertrichter mit Chamberlandeinlage. Ihre Reinigung ließ sich dadurch sehr gut bewerkstelligen, daß der Rückstand mit einem Borstenpinsel und heißem Wasser zunächst entfernt und dann verdünnte, warme Chromschwefelsäure durchgesaugt wurde, was eine Verbrennung der organischen Substanz, die in den Poren haftete, herbeiführte. Mit Ammoniak und Wasser wurde gut nachgewaschen.

Hoppe-Seyler's Zeitschrift f. physiol. Chemie. XL. 
A. Schittenhelm und F. Schröter,

Das Filtrat ${ }^{1}$ ) von der Silberfällung wurde durch Einleiten von Schwefelwasserstoff vom Silber befreit, zur Vertreibung des Ammoniaks eingeengt und in der bis zur vollständigen Klarheit filtrierten Lösung die Nucleinsäure als Kupfersalz gefällt. Resultat positiv.

Portion 2 nach 10 Tagen verarbeitet.

Gefunden: 10,22 mg Gesamtbasen-N.

Nachweis der unzersetzten Nucleinsäure positiv.

Portion 3 nach 15 Tagen verarbeitet.

Gefunden: 7,14 mg Gesamtbasen-N.

Nachweis der unzersetzten Nucleinsäure $\mathrm{schw}$ a ch positiv.

Bacterium coli.

Versuch II angesetzt analog dem Versuch I.

Portion 1 nach 5 Tagen verarbeitet.

Gefunden: 3,1 mg Gesamtbasen-N.

Portion 2 nach 10 Tagen.

Gefunden: $5,7 \mathrm{mg}$ Gesamtbasen-N.

Portion 3 nach 15 Tagen.

Gefunden: $16,3 \mathrm{mg}$ Gesamtbasen-N.

Versuch III. $250 \mathrm{ccm}$ Uschinskysche Lösung (mit Natr. asparaginic. und ammon. lactic.) $+2,5 \mathrm{~g}$ nucleinsaures Natrium wurden mit demselben Stamm von Bact. coli geimpft und 10 Tage lang im Brutschrank stehen gelassen; nachher wie die obigen Versuche verarbeitet.

Gefunden: 20,7 mg Gesamtbasen-N.

Nachweis unzersetzter Nucleinsäure negativ.

Bacterium coli.

Versuch IV wie I.

Portion 1 nach 10 Tagen.

Gefunden: $8,4 \mathrm{mg}$ Gesamtbasen-N.

Nachweis unzersetzter Nucleinsäure positiv.

1) Bei diesem und dem 4. Versuche wurde auf unzersetzte Nucleinsäure nach der Basenfällung geprüft, während es mit allen anderen, ausgenommen II, vor derselben mit einer kleinen Probe geschah. 
Portion 2 nach 15 Tagen.

Gefunden: $5,3 \mathrm{mg}$ Gesamtbasen-N.

Unzersetzte Nucleinsäure spurweise noch vorhanden.

Staphylococcus pyogenes albus.

Versuch V. $1500 \mathrm{ccm}$ Uschinskysche Lösung (sine natr. asparag. und ammon. lact.) $+15 \mathrm{~g}$ nucleinsaures Natrium wurden mit Staphylococcus pyogen. alb., dessen Reinkultur wir eigens hierzu aus dem Eiter einer Fingerphlegmone züchteten, geimpft und in 3 Portionen in den Brutschrank gestellt.

Portion 1 nach 5 Tagen wie die letzten Versuche verarbeitet.

Gefunden: 25,8 mg Gesamtbasen-N.

Nachweis unzersetzter Nucleinsäure negativ.

Portion 2 nach 10 Tagen.

Gefunden: $27,9 \mathrm{mg}$ Gesamtbasen-N.

Nachweis unzersetzter Nucleinsäure negativ.

Portion 3 nach 15 Tagen.

Gefunden: $30,7 \mathrm{mg}$ Gesamtbasen-N.

Nachweis unzersetzter Nucleinsäure negativ.

Versuch VI. $500 \mathrm{ccm}$ Uschinskyscher Lösung (cum natr. asparag. und ammon. lact.) + 5 g nucleinsaures Natrium wurden nach Impfung mit Staphylococcus 10 Tage lang im Brutschrank stehen gelassen und in der beschriebenen Weise verarbeitet.

Gefunden: $32,76 \mathrm{mg}$ Gesamtbasen-N.

Nachweis unzersetzter Nucleinsäure negativ.

Bakteriengemisch aus Faeces.

Versuch VII. $1500 \mathrm{ccm}$ Uschinskyscher Lösung (ohne asparaginsaures Natrium und milchsaures Ammon) $+15 \mathrm{~g}$ nucleinsaures Natrium wurden mit Faecesbakterien geimpft und in 3 Portionen zu je $500 \mathrm{ccm}$ bei Brutschranktemperatur stehen gelassen.

Portion 1 nach 5 Tagen. 
Gefunden: 13,4 mg Gesamtbasen-N.

Nachweis unzersetzter Nucleinsäure positiv. Portion 2 nach 10 Tagen.

Gefunden 21,6 mg Gesamtbasen-N.

Nachweis unzersetzter Nucleinsäure negativ.

Portion 3 nach 15 Tagen.

Gefunden 12,5 mg Gesamtbasen-N.

Nachweis unzersetzter Nucleinsäure negativ.

Versuch VIII. $500 \mathrm{ccm}$ Uschinskyscher Lösung (cum natr. asparagin. und ammon. lactic.) $+5 \mathrm{~g}$ nucleinsaures Natrium wurden mit demselben Bakteriengemisch aus Faeces geimpft und 10 Tage im Brutschrank stehen gelassen; dann wie oben verarbeitet.

Gefunden 19,8 mg Gesamtbasen-N.

Nachweis unzersetzter Nucleinsäure negativ.

Sämtliche Versuche sind der besseren Übersichtlichkeit halber in folgender Tabelle zusammengestellt.

\begin{tabular}{|c|c|c|c|c|c|c|c|c|c|}
\hline \multirow[t]{2}{*}{$\begin{array}{l}\text { Versuch } \\
\text { Nr. }\end{array}$} & \multirow[t]{2}{*}{ Nährlösung } & \multirow[t]{2}{*}{ Geimpft mit } & \multirow{2}{*}{$\begin{array}{c}\text { nuclein- } \\
\text { saures } \\
\text { Natrium } \\
\text { in } \mathbf{g}\end{array}$} & \multicolumn{3}{|c|}{$\begin{array}{c}\text { Ges.-Basen } \mathrm{N} \text { in } \mathrm{mg} \\
\text { nach }\end{array}$} & \multicolumn{3}{|c|}{$\begin{array}{c}\text { Probe auf unzer } \\
\text { setzte Nucleinsäu } \\
\text { nach }\end{array}$} \\
\hline & & & & $\begin{array}{c}5 \\
\text { Tagen }\end{array}$ & $\begin{array}{c}10 \\
\text { Tagen }\end{array}$ & $\begin{array}{c}15 \\
\text { Tagen }\end{array}$ & $\begin{array}{c}5 \\
\text { Tagen }\end{array}$ & $\begin{array}{c}10 \\
\text { Tagen }\end{array}$ & \\
\hline I & Usch. (sine) & Bact. coli & 5 & 1,4 & 10,22 & 7,14 & + & + & \\
\hline II & & & 5 & 6,2 & 11,4 & 33,0 & & & \\
\hline III & Uschinsky & & 5 & - & 41,4 & - & & - & \\
\hline IV & Usch. (sine) & & 5 & 一 & 8,4 & $\tilde{\delta}, 3$ & & + & \\
\hline V & 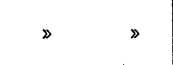 & $\begin{array}{l}\text { Staphylo- } \\
\text { kokken }\end{array}$ & 5 & 25,8 & 27,9 & 30,7 & - & - & \\
\hline VI & Uschinsky & $»$ & 5 & - & 32,76 & - & & - & \\
\hline VII & Usch. (sine) & Faeces & 5 & 13,4 & 21,6 & 12,5 & + & - & \\
\hline VIII & Uschinsky & $»$ & 5 & - & 19,6 & - & & 一 & \\
\hline
\end{tabular}

Versuch II und III waren mit $2,5 \mathrm{~g}$ nucleinsaurem Natrium angesetzt, sind aber des besseren Vergleichs wegen auf $5 \mathrm{~g}$ berechnet worden.

Aus der Tabelle ist ersichtlich, daß sämtliche von uns angewandten Bakterienarten imstande waren, die Nucleinsäure abzubauen, wenn auch die Intensität bei den einzelnen Arten eine verschiedene ist. Vergleicht man z. B. die Versuche I, 
II, IV, V mit Versuch VII, so ergibt sich, daß bei ersteren die Menge der abgespaltenen Basen im Laufe von 5-15 Tagen ansteigt, während bei Versuch VII die Abspaltung etwa am zehnten Tage ihren Höhepunkt erreicht hat und von da ab zu sinken beginnt. Eine Erklärung für diese Beobachtung läßt sich vorläufig nicht mit Sicherheit aufstellen. Erst eingehende Versuche in größerem Umfange werden darüber sicheren Aufschluß geben. Der Unterschied zwischen den einzelnen Resultaten kann seinen Grund in folgenden Möglichkeiten haben:

1. Die Einwirkungsweise der verschiedenen Bakterienarten ist eine verschiedene, indem die einen nur eine Spaltung in Basen etc. hervorrufen, während die anderen auch die Basen noch weiter abzubauen imstande sind.

2. Die verschiedenen Bakterienarten sind mehr oder weniger empfindlich gegen schädigende Bestandteile des Nährbodens (infolge Zersetzung) und bakterielle Exkrete.

3. Die Bakterienarten bauen nicht mit der gleichen Geschwindigkeit die Nucleinsäure ab.

4. Die abgespaltene Basenmenge ist abhängig von der Anzahl der wirksamen Bakterien.

Wollte man den erzeugten Gesamtbasenstickstoff in Milligrammen und die Reaktionsdauer in Tagen in ein Koordinatensystem eintragen, so würden sich der Hauptsache nach vier Reaktionskurven konstruieren lassen.

Die Kurve steigt bis zum Maximum der theroretisch möglichen Basenabspaltung und bleibt dauernd in dieser Höhe d.h. : Die Bakterien sind nur fähig, die Nucleinsäure in ihre Komponenten zu spalten, die Basen aber nicht weiter abzubauen,

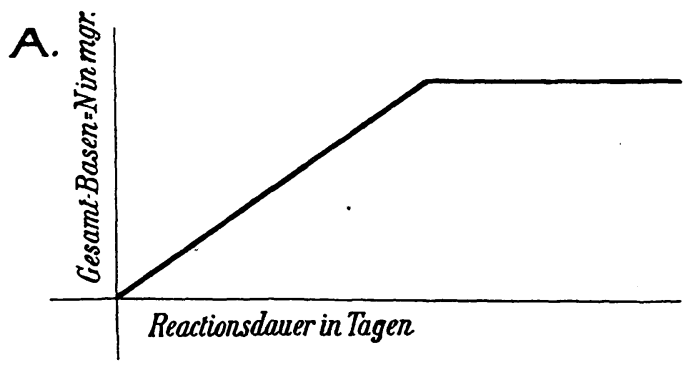
und sind unempfindlich gegen die unter 2 angegebenen Einflüsse. 
Die Kurve steigt bis zum Maximum und sinkt bis zum Minimum d. h.:

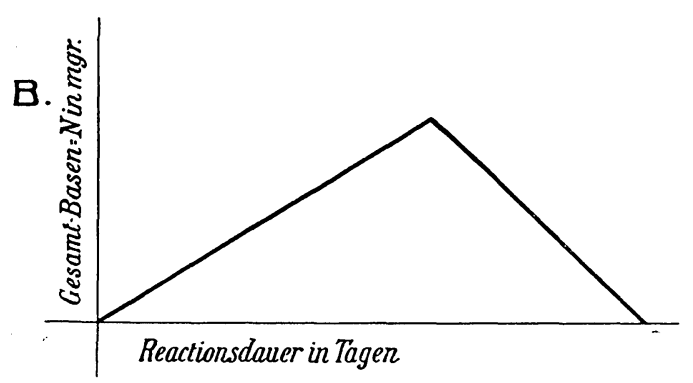

Die Bakterien spalten die $\mathrm{Nu}$ cleinsäure, bauen die Basen weiter ab und sind unempfindlich gegen die sub 2 genannten Einflüsse.

Die Kurve steigt bis zum Maximum, fällt, wird dabei immer flacher und bleibt schließlich in gleicher Höhe d. h.:

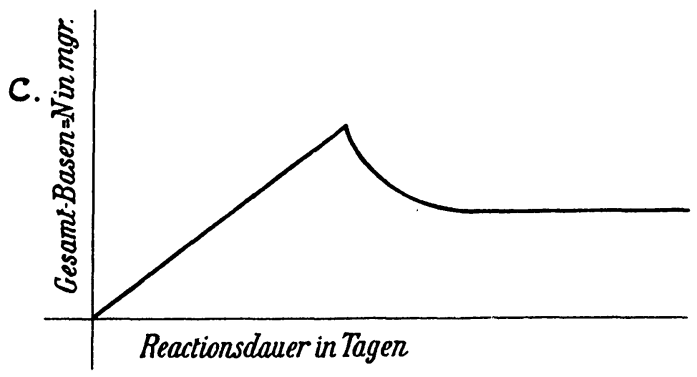

Die Bakterien sind imstande, die Nucleinsäure zu spalten und die. Basen abzubauen, sind aber gegen die durch letzteren

Prozeß erzeugten Produkte empfindlich.

Die Kurve steigt unter allmählichem Flachwerden bis zu einer gewissen Höhe (nicht bis zum theoretischen Maximum!) und hält sich auf dieser d. h.:

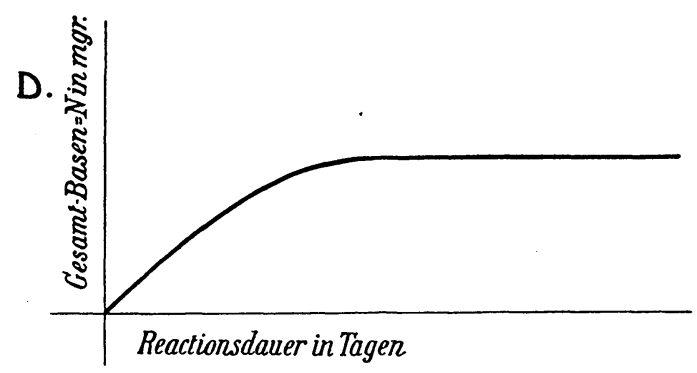

Die Bakterien spalten die Nucleinsäure, sind aber gegen die sub 2 genannten Einflüsse empfindlich. 
Daß die ursprüngliche Zusammensetzung der Nährlösung ${ }^{1}$ ), insbesondere ihr Gehalt an Stickstoff (vgl. Versuch III, VI und VIII) nicht ohne Einfluß auf das Wachstum und die Tätigkeit der Bakterien ist, war wohl vorauszusehen. Bemerkenswert ist aber das Ergebnis der Versuche, welche mit verschiedenen Stämmen derselben Species angesetzt waren (vgl. Versuch I, II und IV). Von den drei verschiedenen Versuchsreihen wurde jede mit einer anderen Reinkultur von Bacterium coli geimpft; alle drei waren jedoch ganz frisch aus Faeces gezüchtet und vor der Verwendung mit den üblichen Methoden auf Säurebildung, Vermögen, Milch zu koagulieren und Zucker zu vergähren, aufs genaueste geprüft. Alle drei Kulturen erfüllten diese Bedingungen in gleicher Weise und dennoch zeigte sich, daß sie in ihrer Wirksamkeit gegen Hefenucleinsäure durchaus kein einheitliches Resultat lieferten.

Endlich bedarf es noch der Erwähnung, daß bei den Versuchen mit Coli und Faeces schon am zweiten Tage lebhafte Gasbildung eintrat, während die Staphylokokken diese Erscheinung nicht aufweisen. Wir kommen auf diese Beobachtung später noch ausführlicher zurück.

$\mathrm{Ob}$ etwa eine nennenswerte Behinderung der Silberfällung durch das Vorhandensein von freier Hefenucleinsäure statthat (analog den Erfahrungen bei der Thymonucleinsäure), haben wir nicht untersucht. Doch müßte dieser Punkt bei umfangreicheren Untersuchungen in Erwägung gezogen werden. Für die vorliegenden Versuche, welche ja gewissermaßen als Vorversuche $\mathrm{zu}$ betrachten sind, ist er nicht von wesentlicher Bedeutung.

1) Vergl. I. Mitteilung, Diese Zeitschr., Bd. XXXIX, S. 205. 